\title{
Unique pharmacological profile of aripiprazole as the phasic component buster
}

\author{
Takashi Hamamura • Toshiki Harada
}

Published online: 9 February 2007

(C) Springer-Verlag 2007

Psychopharmacology (2007)

DOI: $10.1007 / \mathrm{s} 00213-006-0654-2$

In the Conclusions section of the Abstract it should read "aripiprazole is a phasic component buster."

The 11th sentence of the third paragraph should read:

Taken together, under aripiprazole treatment, the "phasic" component is relatively more suppressed than the tonic dopaminergic transmission (phasic component buster).

The online version of this article can be found at http://dx.doi.org/ 10.1007/s00213-006-0654-2.

T. Hamamura $(\bowtie)$

Hamamura Clinic,

5-1-17 Kojima Ogawa Kurashiki,

Okayama 711-0911, Japan

e-mail: ohama@cc.okayama-u.ac.jp

T. Harada

Takahashi Hospital,

2200 Abe Ochiai-cho Takahashi,

Okayama 716-0061, Japan 